\title{
An All-in-One Nanomedicine Consisting of CRISPR-Cas9 and an Autoantigen Peptide for Restoring Specific Immune Tolerance
}

Ying-Li Luo, Li-Fang Liang, Yun-Jiu Gan, Jing Liu, Yue Zhang, Ya-Nan Fan, Gui

Zhao, Anna Czarna, Zi-Dong Lu, Xiao-Jiao Du, Song Shen, Cong-Fei Xu*, Zhe-

Xiong Lian, Jun Wang*

* Corresponding Authors:

mcjwang@scut.edu.cn (Jun Wang), xucf@scut.edu.cn (Cong-Fei Xu)
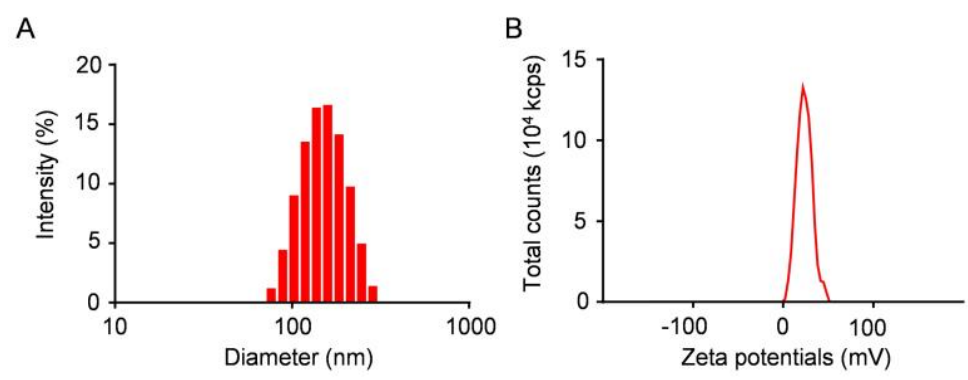

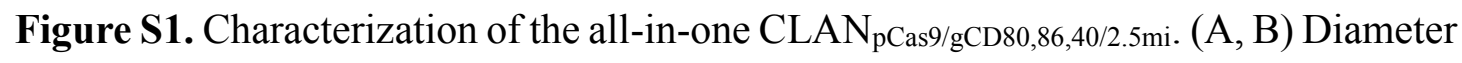

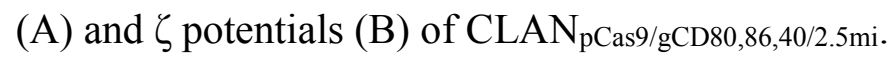




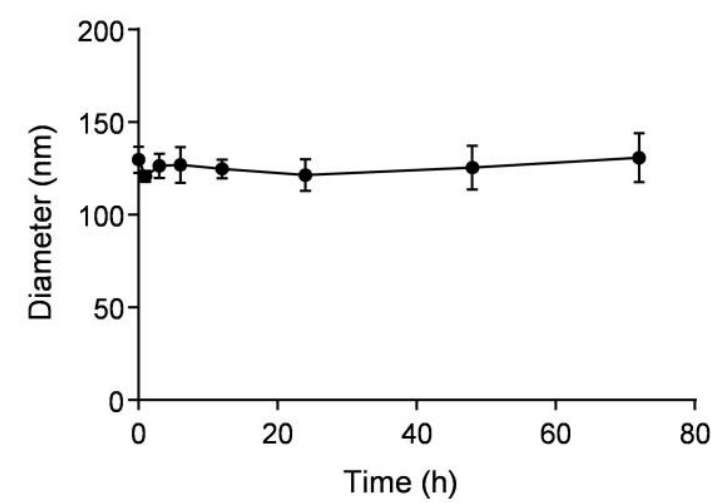

Figure S2. The stability of the all-in-one $\mathrm{CLAN}_{\mathrm{pCas} / \mathrm{gCD} 80,86,40 / 2.5 \mathrm{mi}}$ in DMEM medium containing $10 \% \mathrm{FBS}$. 


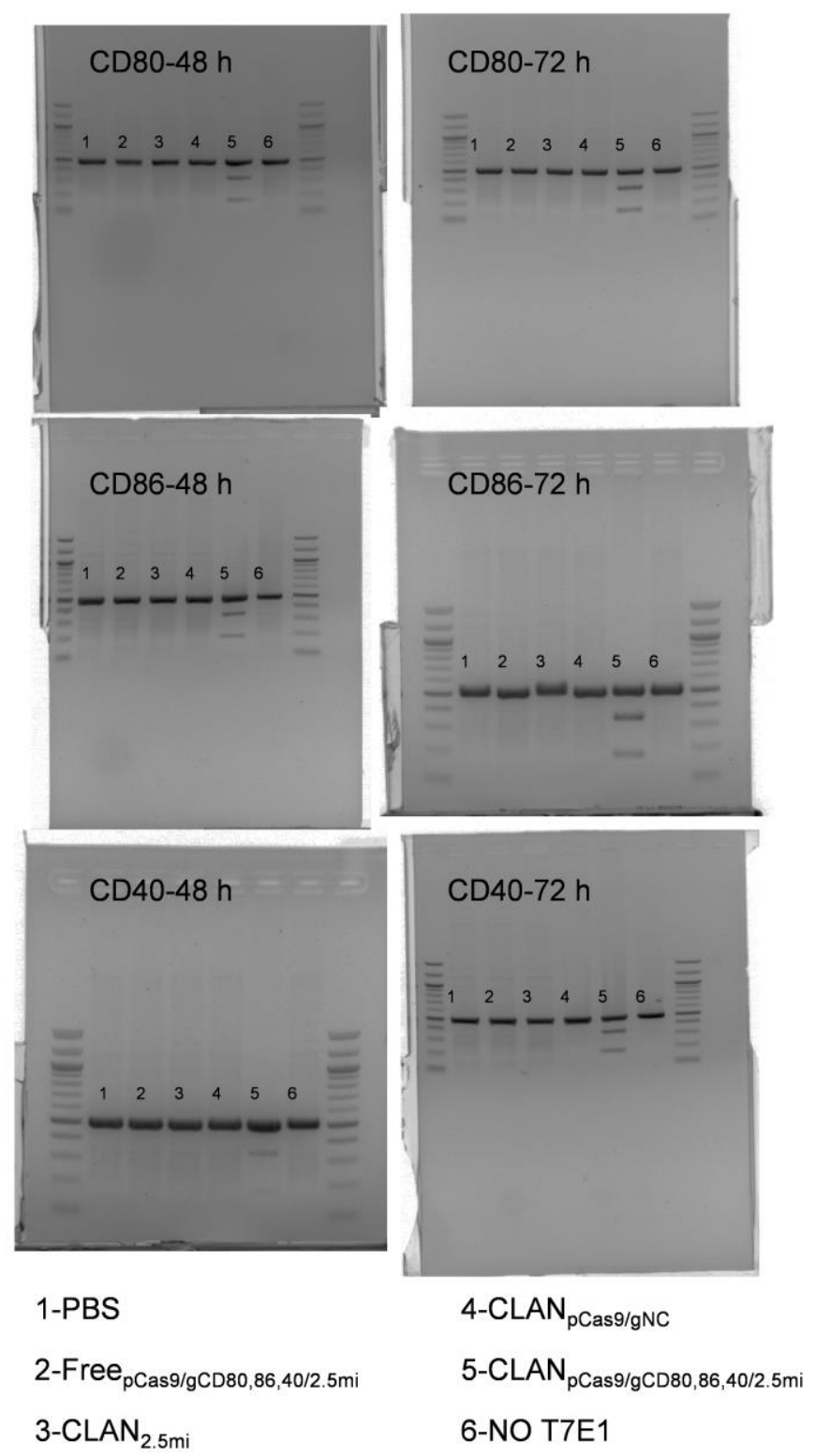

Figure S3. The original uncropped gel images of Figure 1E. 
CD80

Wild type: AAAGTCCGGTTCTTATACTCGGG

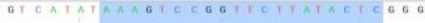

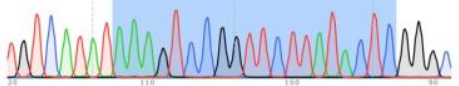

Mutation-1: AAAGTCCGGTTCT. . . . . . CGGG

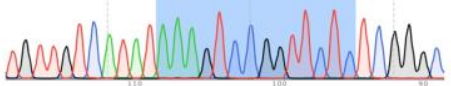

Mutation-2: AAAGTCCGGTTCT- . . . CTCGGG

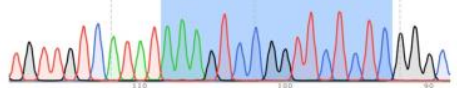

Mutation-3: AAAGTCCGGTTCTTA- - CTCGGG

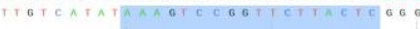

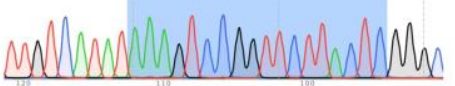

Mutation-4: AAAGTCCGGTTCTTA - GCTCGGG

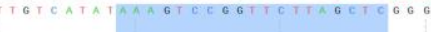

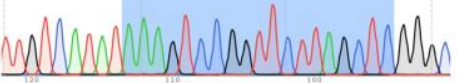

CD86

Wild type: GAAATAAGCTTGCGTCTCCACGG

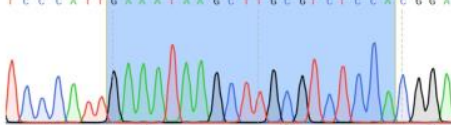

Mutation-1: GAAATAAGCTTGCGT- . . . . CGG

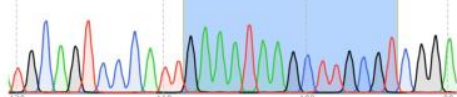

Mutation-2: GAAATAAGCTTGCGTCT- . - CGG

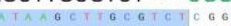

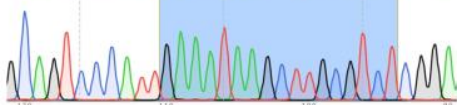

Mutation-3: GAAATAAGCTTGCGTC. . . . . G

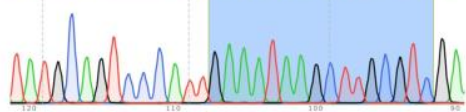

Mutation-4:GAAATAAGCTTGCGTCGTCCACGG

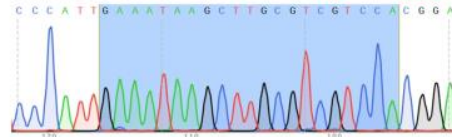

CD40

Wild type: GACAAACAGTACCTCCACGATGG G T G EA G F GACAAACA E TACCOTCCACOEAT OO OC

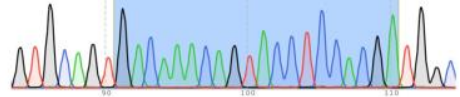

Mutation-1: GACAAACAGTACCTCCA - . - GG

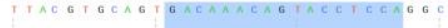

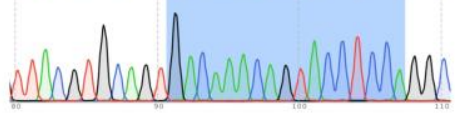

Mutation-2: GACAAACAGTACCTCCA - ATGG

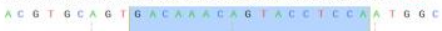

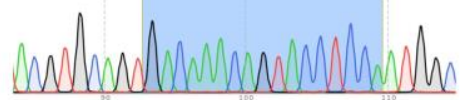

Mutation-3: GACAAACAGTACCTCCA- GATGG

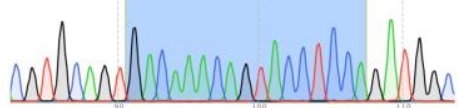

Mutation-4:GACAAACAGTACCTCCAACGATGG

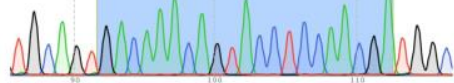

Figure S4. Representative mutated sequences in CD80, CD86 and CD40 genes of

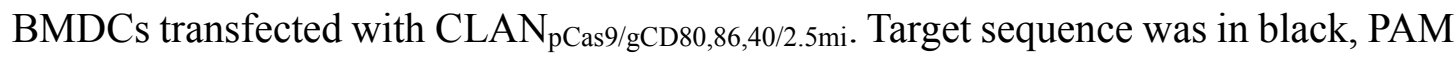
motif was in green and inserted bases were in red. 

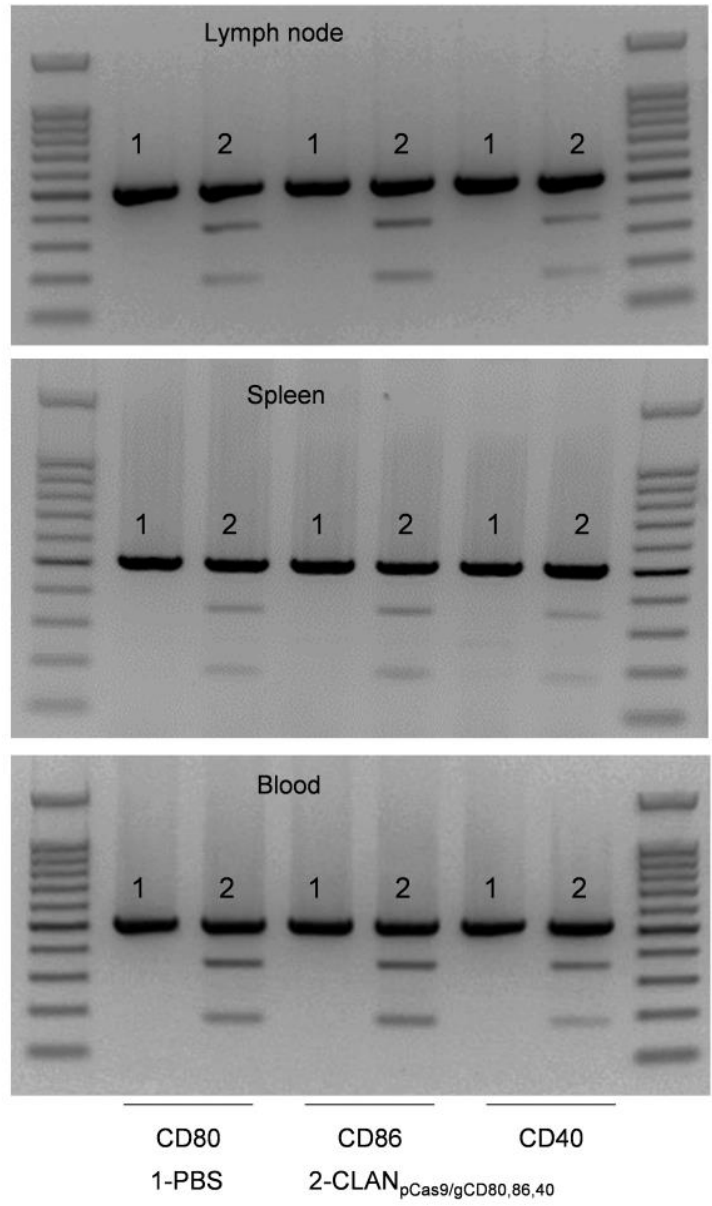

Figure S5. The original uncropped gel images of Figure 3C. 
A

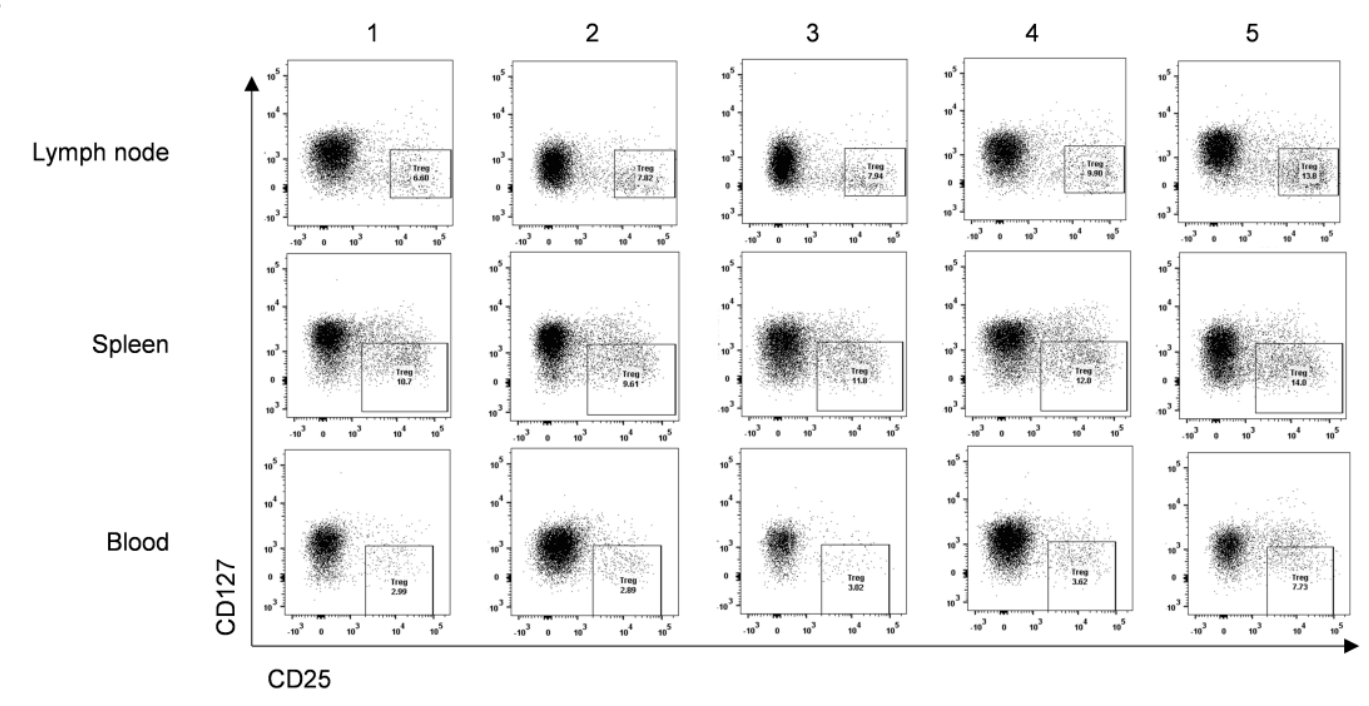

B

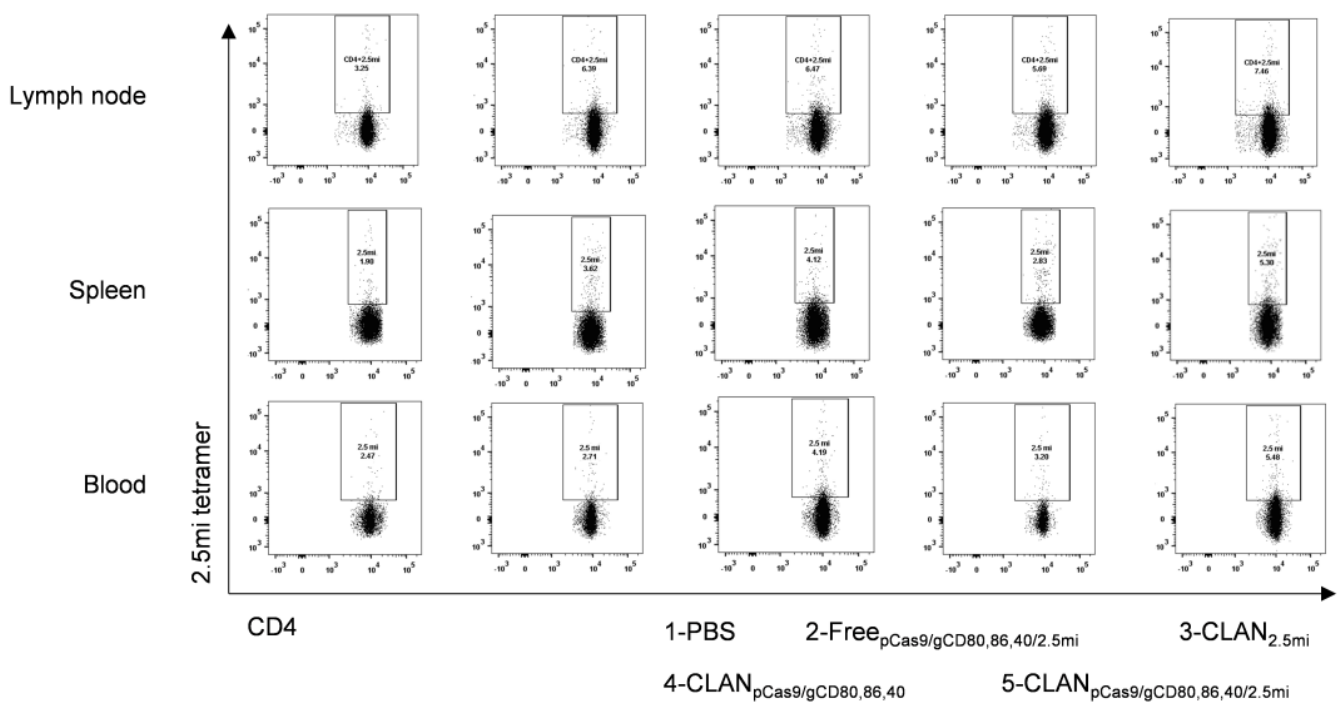

Figure S6. The representative flow cytometry pictures of Figure 4F and G. (A) The percentages of $\mathrm{CD} 25^{+} \mathrm{CD} 127^{\text {low }}$ in $\mathrm{CD} 4^{+} \mathrm{T}$ cells. (B) The percentages of $2.5 \mathrm{mi}$ tetramer ${ }^{+} \mathrm{CD} 4^{+}$in $\mathrm{CD} 4^{+} \mathrm{T}$ cells. 

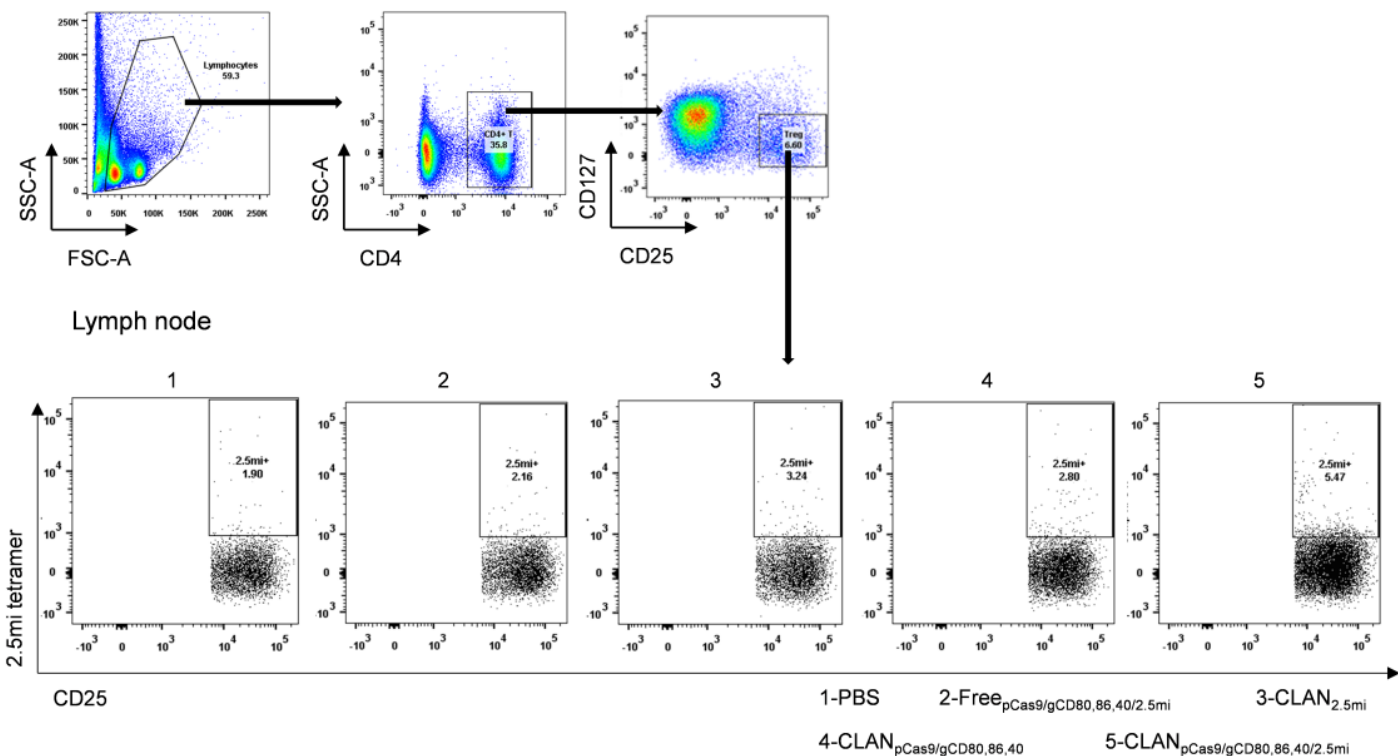

Figure S7. The representative flow cytometry pictures of Figure $4 \mathrm{H}$. The percentages of $\mathrm{CD} 4^{+} \mathrm{CD} 25^{+} \mathrm{CD} 127^{\text {low }} 2.5 \mathrm{mi}$ tetramer ${ }^{+}$Treg cells in lymph node. 
Table S1. Oligos used for cloning gRNA expression vectors.

\begin{tabular}{|c|c|}
\hline Oligos ID & \multicolumn{1}{c|}{ Sequence } \\
\hline CD80 gRNA oligo Forward & 5'-CACCGAAAGTCCGGTTCTTATACTC-3' \\
\hline CD80 gRNA oligo Reverse & 5'-AAACGAGTATAAGAACCGGACTTTC-3' \\
\hline CD86 gRNA oligo Forward & 5'-CACCGAAATAAGCTTGCGTCTCCA-3' \\
\hline CD86 gRNA oligo Reverse & 5'-AAACTGGAGACGCAAGCTTATTTC-3' \\
\hline CD40 gRNA oligo Forward & 5'-CACCGACAAACAGTACCTCCACGA-3' \\
\hline CD40 gRNA oligo Reverse & 5'-AAACTCGTGGAGGTACTGTTTGTC-3' \\
\hline Negative control gRNA oligo Forward & 5'-CACCGCACTACCAGAGCTAACTCA-3' \\
\hline Negative control gRNA oligo Reverse & 5'-AAACTGAGTTAGCTCTGGTAGTGC-3' \\
\hline
\end{tabular}

Table S2. Oligos used for making template for in vitro transcription (IVT).

\begin{tabular}{|c|l|}
\hline \multicolumn{1}{|c|}{ Oligos ID } & \multicolumn{1}{c|}{ Sequence } \\
\hline CD80 gRNA IVT F primer & $\begin{array}{l}5^{\prime} \text { - } \\
\text { TTAATACGACTCACTATAGGGAAAGTCCGGT } \\
\text { TCTTATACTC-3' }\end{array}$ \\
\hline CD86 gRNA IVT F primer & $\begin{array}{l}5^{\prime}- \\
\text { TTAATACGACTCACTATAGGGAAATAAGCTTG } \\
\text { CGTCTCCA-3' }\end{array}$ \\
\hline CD40 gRNA IVT F primer & $\begin{array}{l}5^{\prime} \text { - } \\
\text { TTAATACGACTCACTATAGGGACAAACAGTA } \\
\text { CCTCCACGA-3' }\end{array}$ \\
\hline Negative control gRNA IVT F primer & $\begin{array}{l}\text { 5'- } \\
\text { TTAATACGACTCACTATAGGGCACTACCAGAG } \\
\text { gRNA IVT R primer }\end{array}$ \\
\hline
\end{tabular}


Table S3. Oligos used for T7E1 assay and indels sanger sequencing assay.

\begin{tabular}{|c|c|}
\hline Oligos ID & \multicolumn{1}{c|}{ Sequence } \\
\hline CD80 T7E1 assay F primer & 5'-TTTCCCCATCATGTTCTCCAAA-3' \\
\hline CD80 T7E1 assay R primer & 5'-AATCCTTTTAGTGTCTGCAGAT-3' \\
\hline CD86 T7E1 assay F primer & 5'- ATTGCTGAGGAAGAAAGAGGAG-3' \\
\hline CD86 T7E1 assay R primer & 5'- ACTGACAGTTCTGTTAATGTCT-3' \\
\hline CD40 T7E1 assay F primer & 5'- AGCAGGGACTTTGGAGTGACT-3' \\
\hline CD40 T7E1 assay R primer & 5'- TGACAGACGGTATCAGTGGTCT-3' \\
\hline
\end{tabular}

